\title{
ENDODONTIC IRRIGATION PROTOCOLS: EFFECTS ON THE MICRO-TENSILE BOND STRENGTH OF DIFFERENT ADHESIVE SYSTEMS TO CORONAL DENTIN
}

\author{
Ahmed El-Kabbaney *, Dina S. Farahat ${ }^{* *}$ and Ashraf Ibrahim Ali ${ }^{* * *}$
}

\begin{abstract}
Objectives: The purpose of this study was to evaluate and compare the effect of endodontic irrigation protocols on the micro-tensile bond strength of total-etch and universal adhesives to coronal dentin.

Materials and Methods: Forty teeth were collected and their occlusal surfaces were flattened until middle dentin was exposed. They were divided into four groups according to surface treatment of dentin (untreated (control), $\mathrm{NaOCl}$, EDTA and combination of the two irrigants). Two different adhesives were selected for the current study (total-etch adhesive and universal adhesive). Adhesives were applied according to manufacturers' instructions, then composite restoration was built up incrementally. Resin/dentin specimens were sectioned for micro-tensile bond strength ( $\mu \mathrm{TBS}$ ) testing. A specimen from each group was randomly selected and investigated under scanning electron microscope (SEM).
\end{abstract}

Results: Regarding the total-etch adhesive; the control group showed the highest value of $\mu \mathrm{TBS}$, followed by the group irrigated with $\mathrm{NaOCl}+$ EDTA, EDTA and $\mathrm{NaOCl}$; respectively. Regarding the Universal adhesive; dentin irrigated with a combination of EDTA+NaOCL was found to give the highest value of $\mu$ TBS. It was followed by that irrigated with EDTA and control group. Irrigation with $\mathrm{NaOCl}$ resulted in a dramatic decrease in the $\mu \mathrm{TBS}$ in comparison with the other groups $(\mathrm{p}<0.001)$.

Conclusions: The use of a combination of $\mathrm{NaOCl}$ and EDTA as a regular endodontic irrigants in dental clinics was found to highly improve the quality of bond strength between dentin and both the total-etch and universal adhesives used for bonding the coronal composite restoration.

KEYWORDS: Sodium hypochlorite, Ethylenediamine tetraacetic acid, total-etch adhesive, Universal adhesive, Micro-tensile bond strength.

* Department of Endodontics, Faculty of Dentistry, Mansoura University, Mansoura, Egypt.

** Department of Dental Biomaterials, Faculty of Dentistry, Mansoura University, Mansoura, Egypt.

*** Department of Operative Dentistry, Faculty of Dentistry, Mansoura University, Mansoura, Egypt. 


\section{INTRODUCTION}

Contemporary root canal treatment (RCT) requires not only proper cleaning, shaping and creating adequate apical seal but also it requires the establishment of an effective coronal seal immediately after RCT to avoid coronal microleakage. ${ }^{1}$ Coronal dentin inevitably comes in contact with various endodontic instruments, medications and intracanal irrigants during chemo-mechanical debridement protocols. These chemicals used in root canal preparation, may alter the structure of the surface of coronal dentine available for the coronal restoration and modify its bonding ability to the restorative material. ${ }^{2}$

Among common daily used irrigants are sodium hypochlorite $(\mathrm{NaOCl})$ solution and ethylenediamine tetraacetic acid (EDTA). The biomechanical properties of dentin can be changed due to the usage of these irrigant solutions. The properties of inorganic and organic components, hardness, permeability and solubility of dentin are altered by changes in the calcium/phosphate ratio. ${ }^{3,4}$ Because of the successful removal of the smear layer and enhanced dentin permeability, consecutive root canal irrigation with $\mathrm{NaOCl}$ followed by EDTA was shown to maximize the antisepsis effectiveness. ${ }^{5}$ Hence, it can be assumed that endodontically treated teeth receiving $\mathrm{NaOCl}$ and EDTA will react differently to the adhesive process for coronal restoration, inducing new challenges to adhesive bond regarding its strength and durability. $\mathrm{NaOCl}$ has been found to be cytotoxic in a number studies, as well as having the potential to weaken the dentin-resin bond. ${ }^{6,7}$ The main rationale could be corroborated to $\mathrm{NaOCl}$ 's oxidizing characteristics, which can result in an oxygen rich layer that if formed in dentin, can hinder the polymerization of resin material, thus resulting in microleakage. ${ }^{3,8,9}$

Furthermore, RCT eliminates the moisture of the dentin structure, a change which could influence the adhesive bond strength. In addition, compressive and tensile strengths of dentin in pulpless teeth seem to be controversially different from normal dentin with lower Young's modulus and proportional limit values recorded in compression tests ${ }^{8}$.

For efficacious elimination of the inorganic and organic constituents of the smear layer, root canal irrigation with $10 \mathrm{ml}$ of $17 \%$ EDTA, followed by $10 \mathrm{ml}$ of $5 \% \mathrm{NaOCl}$ has been suggested. ${ }^{10}$ Due to the efficient removal of the smear layer and the enhanced dentin permeability, the sequential irrigation strategy with $\mathrm{NaOCl}$ and EDTA was found to maximize the efficiency of antisepsis. ${ }^{11}$ The coronal dentin surface is eroded by successive deproteinizing $\mathrm{NaOCl}$ and decalcifying EDTA treatments, resulting in a normal hybrid layer. ${ }^{12}$ Another reason to use a mixture of these irrigants can be attributed to the fact that chelating solutions like EDTA tend to remove calcium ions from the dentin surface, exposing the collagen matrix, which may lead to bacterial adherence (e.g. Enterococcus Faecalis) in cases of recontaminations. ${ }^{13}$

The objective of this research is to assess the impact of using sodium hypochlorite \& ethylenediamine tetraacetic acid root canal irrigants on the micro-tensile bond strength of both self-etch and universal adhesives to coronal dentin. This study was designed to test the null hypothesis that, neither the irrigant solutions nor the used adhesive systems would affect the micro-tensile bond strength of these adhesives to coronal dentin.

\section{MATERIALS AND METHODS}

\section{Materials}

In this study two different types of adhesives were used, Total-Etch Dental Adhesive System; OptiBond $^{\mathrm{TM}}$ FL (Kerr, Orange, CA, USA) and Universal Dental Adhesive System; OptiBond ${ }^{\mathrm{TM}}$ Universal (Kerr, Orange, CA, USA). A universal nano-hybrid resin composite was used as the restorative choice of material; Herculite ${ }^{\mathrm{TM}}$ Ultra 
(Kerr, Orange, CA, USA). In addition, two types of root canal irrigants were used in this study; commercially used sodium hypochlorite of $5.25 \%$ concentration ( $\mathrm{NaOCl}$ ) (AK Medical Solution, Khalaf Pharm, Egypt) as an irrigant solution and Ethylenediamine tetraacetic acid (17\% EDTA) (AK Medical Solution, Khalaf Pharm, Egypt) as an irrigant solution. A LED curing unite (Demi ${ }^{\mathrm{TM}}$ Plus, Kerr, Orange, CA, USA) with a light intensity of $800 \mathrm{~mW} / \mathrm{cm}^{2}$ was used. A radiometer (Demetron Research Corp., Danbury, CT, USA) was regularly used to monitor the light intensity.

\section{Methods}

\section{Teeth Selection}

A total of forty human caries free third molar teeth were selected for the current study. They were obtained following the protocol reviewed and approved by the Ethical Committee, Faculty of Dentistry, Mansoura University, Mansoura, Egypt. After acquiring signed consents from patients, the teeth were harvested at the Oral Surgery clinic, Faculty of Dentistry, Mansoura University where they were scheduled for surgical impaction removal. The storage protocols followed international and institutional infection control guidelines. An ultrasonic scaler was used to remove all the external debris and distilled water was used as a storage medium for the specimens and was replaced every 2 days. A stereomicroscope (Leica, Hanau, Germany) was used to examine the teeth at a magnification of $\mathrm{x} 45$ to exclude carious teeth or any teeth with defects or cracks. After examination, the teeth were stored at $4{ }^{\circ} \mathrm{C}$ in an antibacterial $0.2 \%$ thymol solution until needed, for a period that did not exceed 3 months.

\section{Preparation of specimens}

A rigid polyvinyl chloride (PVC) cylinder (40 $\mathrm{mm}$ height and $15 \mathrm{~mm}$ diameter) was filled with chemically-cured acrylic resin blocks (Acrostone,
Anglo-Egyptian Company, Cairo, Egypt) at the dough stage. The teeth were centrally fixed in chemically-cured acrylic resin blocks, $1 \mathrm{~mm}$ apical to the cemento-enamel junction.

The resin blocks with teeth were fixed in an automated low speed precision saw (Isomet4000, Buehler Ltd, LakeBluff, IL, USA). The crown length was measured from the cusp tips to the cementoenamel junction by caliper. After marking the midway distance, the teeth were sectioned to remove the occlusal surface at the middle of the crown. Dentin surfaces were planed and polished using serial silicon carbide sandpapers (\#400 grit- \#600 grit) under water cooling to produce standardized smear layers. The dentin is exposed, as identified by observation of the pulp horn by transparency and x-ray.

The prepared specimens were divided randomly into four experimental groups $(n=10)$ according to the type of surface treatment applied to prepared dentin.

Group 1: The prepared surfaces were treated with deionized water (control group).

Group2: The prepared surfaces were treated with $5.25 \%$ commercially used sodium hypochlorite solution $(\mathrm{NaOCl})$ for 30 minutes refreshed every 10 minutes.

Group3: The prepared surfaces were treated with 17\% EDTA for 5 minutes followed by washing using deionized water for 10 seconds.

Group4: The prepared surfaces were treated with $5.25 \% \mathrm{NaOCl}$ for 30 minutes and then treated with 17\% EDTA for 5 minutes and rinsed with deionized water for 10 seconds in order to simulate the period of time the dentin is in contact with endodontic irrigants.

Each of the previous groups was further subdivided into two subgroups $(\mathrm{n}=5)$ according to the adhesive system applied, total-etch adhesive system (subgroup A) and universal adhesive (subgroup B). 
After application of adhesives and resin composite build up according to manufacturer instructions, one tooth from each subgroup was selected for scanning electron microscope (SEM), while the rest were subjected to micro-tensile bond strength testing.

Subgroups (A) $n=5$, were bonded with total-etch adhesive according to the manufacturer's instructions; the dentin surface was etched with $37 \%$ phosphoric acid for 15 seconds then rinsed for 15-30 seconds. The dentin surface was dried using a cotton pellet followed by application of a primer for 15 seconds then gentle air-blowing for 5 seconds. After that, the bonding agent was applied for 30 seconds followed by gentle air-blowing for 5 seconds. The adhesive was then light cured with the LED curing unit.

Subgroups (B) $n=5$, were subjected to the universal adhesive with self-etch mode of bonding according to the manufacturer's instructions. The dentin surface was dried followed by employing the adhesive to the dentin surface for 20 seconds. Excess adhesive was dispersed by oil / moisture-free compressed air until a glossy, immobile film layer resulted. Avoiding pooling was taken into consideration, since this can compromise the fitting accuracy of the restoration. The adhesive was then light cured with a LED curing unite.

A customized metallic square mold $(4 \mathrm{~mm}$ $\times 4 \mathrm{~mm}$ ) was utilized in the composite build up process on the prepared surface of dentin of the selected teeth. Two increments of composite, with a thickness of $2 \mathrm{~mm}$, were successively applied to the dentin bonded surface and light cured individually for 20 seconds. When the build-up was accomplished; a square piece of sticky paper of $3 * 3$ $\mathrm{mm}^{2}$ was placed on the center of each tooth. A red colored permanent marker was used to delineate the peripheral area around the sticky paper. After removal of the sticky paper, a square was drawn in the center of the occlusal surface of the composite using a permanent green marker. This color-coded marking facilitated the differentiation and selection of central bonded beams from peripheral ones.

Thermal fatigue method was used to evaluate durability of bond by simulating the thermal fluctuations that occur in the oral cavity during eating / drinking. The effect of thermocycling could be due to water heating accelerating the effects of hydrolysis on the interface. The bonded specimens in each group were thermocycled (SD Mechatroniks thermocycler, Germany) between $5^{\circ} \mathrm{C}$ and $55^{\circ} \mathrm{C}$ for 500 cycles. The dwell time in each bath was 20 seconds and the transfer time between the two baths was 5 seconds.

\section{Micro-tensile bond strength testing}

Beam (stick) specimens with dimensions of $0.9 \mathrm{x}$ $0.9 \times 5.5 \mathrm{~mm}$ were sectioned from restored teeth to measure the micro-tensile bond strength of the two adhesive systems to dentin. The precision saw was used in the longitudinal sectioning of the restored specimens across the bonded interface in both " $\mathrm{x}$ " and " $\mathrm{y}$ " directions to obtain beams formed of composite and dentin with adhesive at the interface.

A custom-made gripping attachment was employed to fix acrylic blocks with mounted teeth rigidly in place and aligned with the sectioning direction. This positioned the cutting disc at a right angle to the occlusal surface and ensured the longitudinal sectioning to be perpendicular to the flat occlusal surface of restored teeth. The attachment had an Lshape and was formed of a cylindrical metal ring (16-mm in diameter, 3-mm thickness, and 2-mm height) connected at its base to a metallic rod that enabled mounting of the attachment into the saw machine. To guarantee the precise placement and ease of rotation of the acrylic blocks inside the gripping attachment, two axial and perpendicular groves were made on the upper surface of the metallic ring.

After mounting, a $0.3-\mathrm{mm}$ thick diamond coated wafering blade (Buehler, IL, USA), with 
water cooling system was used to serially section the restored teeth at $2050 \mathrm{rpm}$ and a $8.8 \mathrm{~mm} / \mathrm{min}$ feeding rate. The cutting was done first in a buccolingual direction then in a mesio-distal direction by rotating the specimens $90^{\circ}$ clockwise. Finally, beams were acquired by a horizontal cut that was done at the cemento-enamel junction. The dimensions of each beam were verified using a digital caliper (Absolute Digimatic, Mitutoyo, Tokyo, Japan) to the nearest $0.01 \mathrm{~mm}$ and the cross-sectional area for each beam was determined to be used in calculation of the actual bond strength values. Then, each beam was kept at room temperature in artificial saliva in a tightly sealed plastic cone with labels that showed the tooth of origin and subgroup.

Twenty beams were tested for each subgroup. A universal testing machine (Instron, MA, USA) with a load cell of $500 \mathrm{~N}$ (Figure 12) was used to measure the $\mu$ TBS. Each beam was individually positioned in the central groove of Geraldeli's jig and fixed in place using cyanoacrylate-based glue (Zapit, DVA Inc, USA). The jig was mounted in the machine and the beams were subjected to tensile load at a crosshead speed of $0.5 \mathrm{~mm} / \mathrm{min}$, till failure of bonding. $\mu$ TBS was calculated in Megapascal (Bluehill Lite software, Instron, MA, USA). Two-way ANOVA test followed by one- way ANOVA and unpaired Student's t-test were used to statistically analyze the obtained results.

\section{Scanning electron microscopy}

A random specimen from each subgroup was selected for SEM. to remove the debris over the restoration and dentin margins, 10\% orthophosphoric acid was used to clean the specimens for five seconds. Specimens then were mounted on aluminum stubs using a double-sided conductive copper tape, then sputter coated by gold then mounted to SEM (JEOL JSM 6510 lv, Japan) and investigated at an accelerated voltage of $20 \mathrm{kv}$ at a magnification of $1500 X$.

\section{RESULTS}

Two-way ANOVA test was used to assess the overall significant effect of the different variables on the outcome of this study. The two variables under investigation (adhesives and endodontic irrigants) had a statistically significant effect on dentin $\mu$ TBS ( $p<0.001$ ), according to results of the two-way ANOVA. The interaction between the types of adhesives used and the endodontic irrigants showed a statistically significant difference $(p<0.001)$. Table (1), Fig (1)

TABLE (1): Results of two-way ANOVA test.

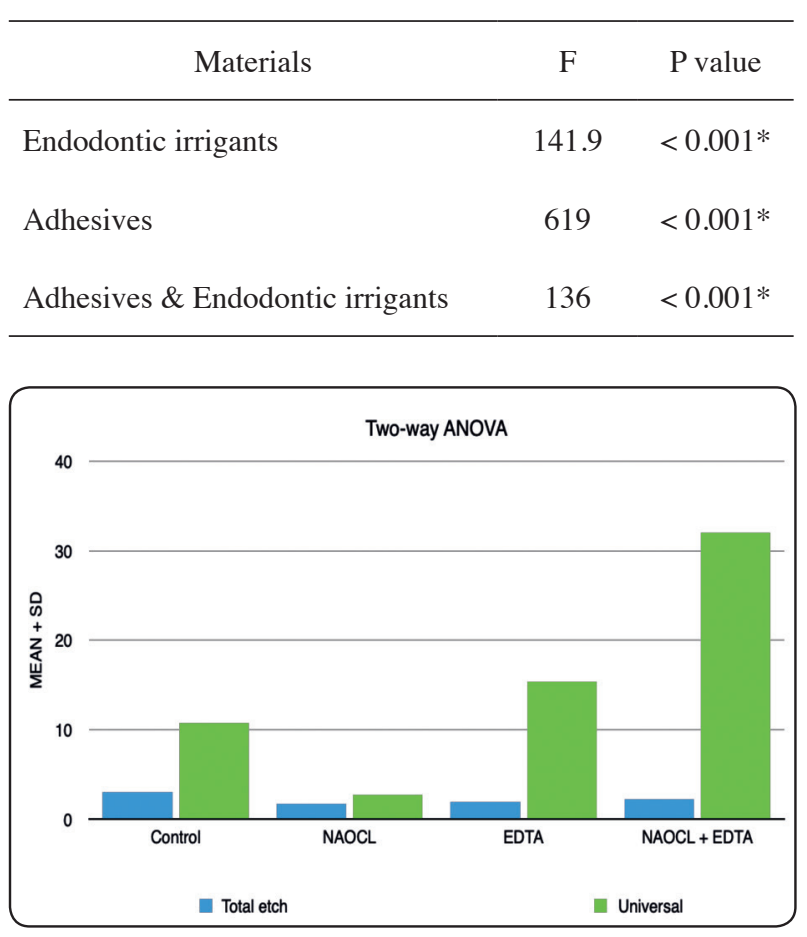

Fig. (1) Graph summarizing 2-way ANOVA results

The values were further analyzed through oneway ANOVA test. Regarding the total-etch adhesive; the control group showed the highest value of $\mu$ TBS (2.99), to be followed by group irrigated with $\mathrm{NaOCl}+$ EDTA (2.21), EDTA (1.85) and $\mathrm{NaOCl}$ (1.66); respectively. The control group revealed a significant difference in $\mu$ TBS with all the tested 
groups $(\mathrm{p}<0.001)$. Dentin treated with a combination of $\mathrm{NaOCl}$ and EDTA showed a significant improvement in the $\mu$ TBS $(\mathrm{p}<0.001)$ when compared with that irrigated with $\mathrm{NaOCl}$, whereas there were no significant differences $(\mathrm{p}<0.05)$ between groups irrigated with EDTA and $\mathrm{NaOCl}$. Also, irrigation with the combination of $\mathrm{NaOCl}$ and EDTA didn't significantly increase $(\mathrm{p}<0.05) \mu \mathrm{TBS}$ than EDTA. Table (2), Fig (2)

Regarding the Universal adhesive; dentin irrigated with a combination of EDTA $+\mathrm{NaOCL}$ (32.01) was found to give the highest value of $\mu$ TBS. It was followed by groups irrigated with EDTA (16.01) and control group (11.09). Irrigation

TABLE (2): Comparison between effects of dentin irrigating solutions on $\mu$-tensile bond strength of dentin bonded with self-etch or universal adhesives.

\begin{tabular}{|c|c|c|c|c|c|c|}
\hline & & \multicolumn{4}{|c|}{ Subgroup } & \multirow[t]{2}{*}{$\mathrm{P}$} \\
\hline & & Control & $\mathrm{NaOCL}$ & EDTA & $\mathrm{EDTA}+\mathrm{NaOCL}$ & \\
\hline \multirow{5}{*}{$\begin{array}{l}\text { Total- etch } \\
\text { Adhesive }\end{array}$} & Mean & 2.99 & 1.66 & 1.85 & 2.21 & \multirow{5}{*}{$<0.001 *$} \\
\hline & $\pm \mathrm{SD}$ & 0.73 & 0.44 & 0.47 & 0.55 & \\
\hline & $\mathrm{P} 1$ & & $<0.001 *$ & $<0.001^{*}$ & $<0.001 *$ & \\
\hline & $\mathrm{P} 2$ & & & 0.7 & $0.015^{*}$ & \\
\hline & P3 & & & & 0.19 & \\
\hline \multirow{5}{*}{$\begin{array}{l}\text { Universal } \\
\text { Adhesives }\end{array}$} & Mean & 11.09 & 3.11 & 16.01 & 32.01 & \multirow{5}{*}{$<0.001 *$} \\
\hline & $\pm \mathrm{SD}$ & 2.69 & 0.77 & 4.01 & 8.02 & \\
\hline & $\mathrm{P} 1$ & & $<0.001^{*}$ & $0.01 *$ & $<0.001 *$ & \\
\hline & $\mathrm{P} 2$ & & & $<0.001 *$ & $<0.001 *$ & \\
\hline & $\mathrm{P} 3$ & & & & $<0.001 *$ & \\
\hline
\end{tabular}

P: Probability *: significance $<0.05$

P1: significance relative to control group

P3: significance relative to EDTA

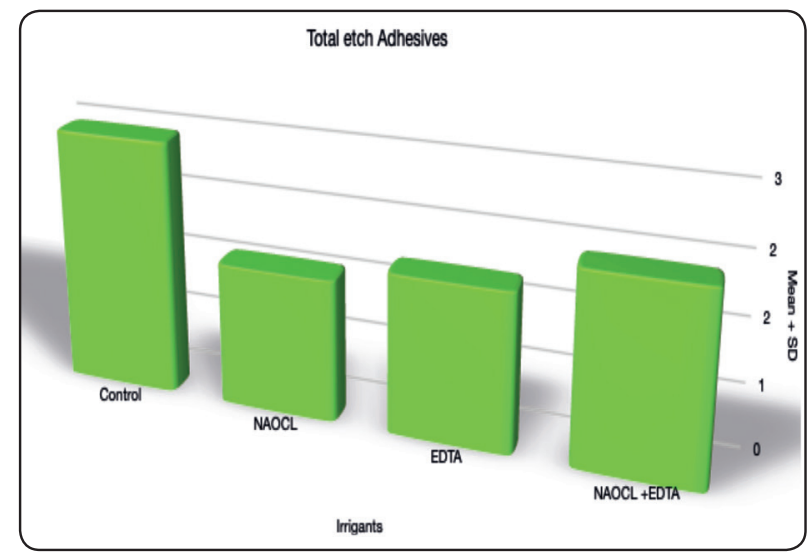

Fig. (2) Graph summarizing one-way ANOVA results for the total-etch adhesive.
Test used: one-way ANOVA test followed by post-hoc tukey

P2: significance relative to $\mathrm{NAOCL}$

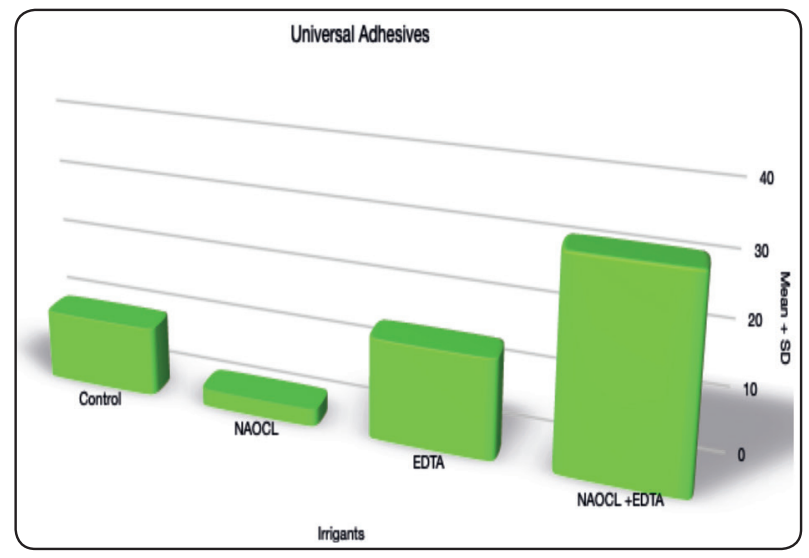

Fig. (3): Graph summarizing one-way ANOVA results for the universal adhesive. 
with $\mathrm{NaOCl}(3.11)$ resulted in dramatic decrease in the $\mu \mathrm{TBS}$ in comparison with the other groups $(p<0.001)$. Groups irrigated with EDTA or its combination with $\mathrm{NaOCl}$ showed statistically significant $(\mathrm{p}<0.001)$ increase in $\mu$ TBS values compared to the control group. Combination of both irrigants was also found to be statistically significant to the group treated with EDTA in a positive way $(\mathrm{p}<0.001)$. Table (2), Fig (3)

Student's t-test was carried out to compare the performance of the used adhesives in each of the endodontic irrigants groups. It was revealed that, regardless the irrigating solutions, bonding with total-etch adhesive has a statistically significant ( $\mathrm{p}<0.001)$ deteriorating effect on the $\mu$ TBS values when compared to bonding using the universal adhesive (self-etch mode). By using $\mathrm{NaOCl}$ as an irrigant, bonding with total-etch adhesive or universal adhesive (self-etch mode) resulted in significant decrease in the $\mu$ TBS. However, total-etch adhesive showed slight decrease in bond strength than the universal adhesive (self-etch mode).

\section{Scanning electron microscopic observations}

The ultrastructural observations of the resin composite/dentine interfaces are illustrated in the scanning electron micrographs shown in (Figures 4, 5). The findings of this study showed true hybridization with scanty short resin tag in case of irrigation with EDTA (Figure 4) and combination of EDTA/ $\mathrm{NaOCL}$, while the control group showed moderate hybrid layer formation. The group irrigated with NaOCL (Figure 5) showed inhomogeneous thin hybridization with short funnel shaped resin tags. The sample irrigated with $\mathrm{NaOCL}$ showed the inner side of the hybrid layer and interrupted (separated hybrid layer and voids) adhesive/dentin interface. The dentinal tubules were completely opened with EDTA and its combination with NaOCL. Irrigation with $\mathrm{NaOCL}$ and control group caused partial obturation of the dentinal tubules.

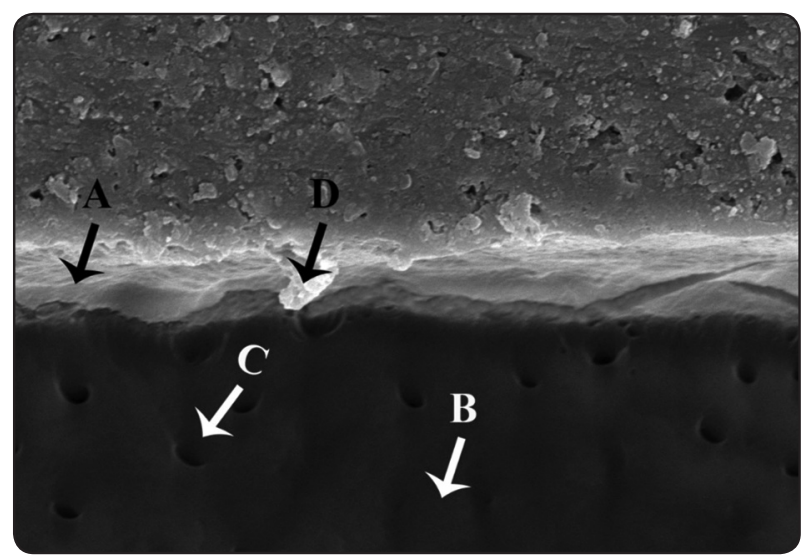

Fig. (4): SEM image of EDTA treated dentin bonded with universal adhesive; A) Relatively thick hybrid layer. B) Complete removal of smear layer. C) Open dentinal tubules. D) Scanty short resin tags.

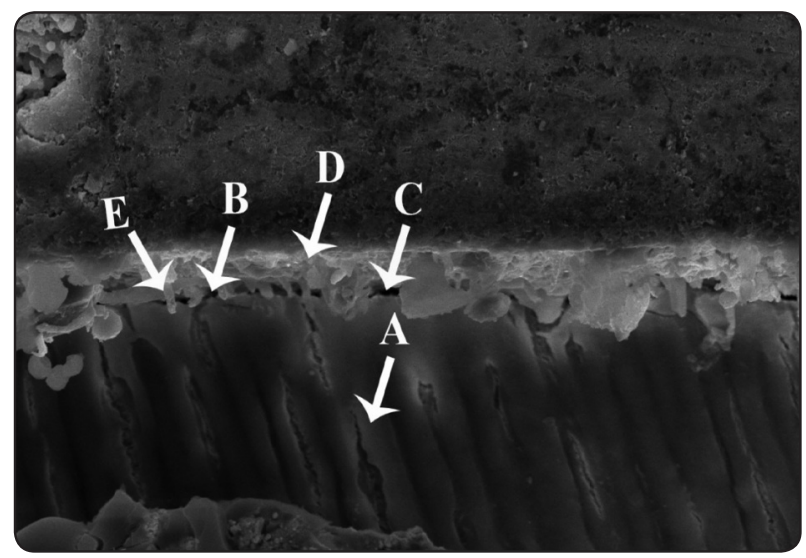

Fig. (5): SEM image of $\mathrm{NaOCl}$ treated dentin surface bonded with total-etch adhesive; A) partial obturation of dentinal tubules. B) micro-gaps. C) Smear layer separation. D) Thin hybrid layer. E) Scanty funnel shaped resin tags.

\section{DISCUSSION}

Frequent observations of clinical cases presented in dental clinics show that endodontic therapy has been a major percentage of the cases. That imposed researchers to study the different factors included in this therapy that could influence the performance of final restorations. The multi-procedures therapy; devitalizing, cleaning and shaping and obturation; is widely considered as a weakening procedure due to the water loss and loss of collagen cross-linking and the removal of tooth structure. ${ }^{14}$ 
However, some studies have found that the moisture content in vital teeth did not differ from that in endodontically treated teeth. ${ }^{15}$ Furthermore a study was undertaken to assess the impact of endodontic treatment and moisture content on various human dentin mechanical properties. The results contradicted the theory that dehydration after endodontic treatment per se weakens the tensile and compressive strengths of the dentin structure. On the other hand, other mechanical properties of pulpless treated teeth, may not be as similar to those of normal vital teeth. ${ }^{16}$

Apart from the factor of water loss associated with RCT, the various irrigants and medicaments used should be kept in mind. These materials help achieve the RCT goal which is an environment devoid from bacteria and well mechanically prepared to receive a restoration. Nevertheless, during chemo-mechanical root canal preparation these materials unavoidably come in contact with dentin of the access cavity, which might alter its properties and compromise its bonding to coronal restorations. ${ }^{17-20}$

In the current study, the occlusal surfaces of the teeth were cut at the middle third to uncover the part of dentin that clinically is exposed to various irrigants and medicaments. The teeth were subjected to different irrigants and then restored by resin composite simulating the steps clinically achieved by dentists. The purpose of the thermocycling of the specimens was to mimic the thermal variations that occur in the oral cavity as a result of drinking, eating, and breathing.

In order to have a complete understanding of the functional performance of adhesives, variable strength tests were introduced. In this study, a micro-tensile strength was used due to its benefits including conservative use of teeth since multiple micro-specimens sectioned from a single tooth could be used at the same time, better control of regional differences e.g. peripheral versus central dentin and enhanced stress distribution at the true interface which helps avoid cohesive failure in both composite and tooth substrate. ${ }^{21}$
Despite these benefits, one study found out that peripheral specimens showed lower $\mu$ TBS to adhesives when compared to centrally located specimens. ${ }^{22}$ On the contrary, another study reported that $\mu \mathrm{TBS}$ to peripheral dentin is higher than for the central specimens. ${ }^{23}$ Taking into account this disparity in regional features of the substrate and to avoid any bias in results of the $\mu \mathrm{TBS}$, a square shape with dimensions $3 \times 3 \mathrm{~mm}$ was marked in the center of the composite-tooth complex with a permanent green color while its peripheral part was marked with permanent red color before sectioning, then the bonded beams from each tooth was divided into peripheral beams and central beams and each group contains an equal number of the central beams.

In either the total-etch adhesive or the universal bonded dentin groups, the $\mathrm{NaOCl}$ variable was found to cause lower values of $\mu \mathrm{TBS}$. This could be attributed to Its strong oxidizing action of dentin matrix components resulting in presence of freeradical at the polymerizing resins tags/dentinal tubule interface. ${ }^{17,18}$ It creates nanometric pores within inter-tubular dentin which are difficult to be infused by resin monomer leading to deficient adhesive interface. ${ }^{24-26}$ Leading to generation of voids in the adhesive/dentin interface. These voids could interfere in the resin infiltration inside the dentinal tubules as well as in the inter-tubular dentin, a case of the present study. It was found that the hybrid layer showed inhomogeneous thin hybridization with short funnel shaped resin tags. Samples irrigated with $\mathrm{NaOCL}$ showed the inner side of the hybrid layer and interrupted (separated hybrid layer and voids) adhesive/dentin interface. Irrigation with $\mathrm{NaOCL}$ caused partial obturation of the dentinal tubules.

In addition, when sodium hypochlorite comes in contact with dentin, it breaks down into sodium chloride and oxygen. As a result, free oxygen on the dentin surface may adversely affect the bond strength by preventing the resin adhesives from full polymerization. It was suggested that a concentration of $5.25 \% \mathrm{NaOCl}$ was to decrease 
dentin young's modulus and its flexing stress. ${ }^{24}$ The flexing stress is reduced by using $2.5 \% \mathrm{NaOCl}$ but the elasticity modulus does not change. ${ }^{27}$ Other studies found that 15 minutes of $1 \% \mathrm{NaOCl}$ solution irrigation significantly reduced the microhardness of root canal dentin at 500 micron and 1000 micron from the pulp-dentin interface. ${ }^{28} \mathrm{In}$ general, $\mathrm{NaOCl}$ application reduced the bond strength values of the adhesive systems and root canal dentin. ${ }^{29}$

The EDTA parameter in the groups bonded with total-etch adhesive was found to weaken the bond strength when compared to the control group. Also, there was no difference between EDTA's results when compared to NaOCl's results. This could be attributed to; although EDTA doesn't denature collagen, its application together with acid etchant, synergizes the erosion occurring on the dentin wall. ${ }^{30,31}$ A nanostructure study revealed that after EDTA treatment there was reduction in stiffness in both peritubular and intertubular dentin thereby adhesion force was reduced..$^{32}$ Nevertheless, given the results of our study, it appears rational to pursue further investigation of the bond strength of totaletch adhesive systems to coronal dentin with regards to the effect of using different endodontic irrigants.

The combination of $\mathrm{NaOCl}$ and EDTA in totaletch group is found to decrease the values of bond strength in comparison to the control group, while this combination is found to strengthen the bond in the universal adhesive group. Lowered bond strength, compared to the control group, could be due to erosion occurring on the dentin after applying both EDTA, $\mathrm{NaOCl}$ and $37 \%$ phosphoric acid etchant.$^{33}$ In addition to this erosion, the oxidizing effect of $\mathrm{NaOCl}$ could decrease the bond strength of different adhesive systems. ${ }^{34}$

The superior bond strength values obtained when dentin was irrigated with a combination of EDTA and $\mathrm{NaOCL}$ in total- etch group may be explained by the sum of etching effects of the irrigants and the etchant. EDTA is a weak acid that can gently etch dentin with subsequent deproteinization carried out with sodium hypochlorite, followed by the etching with $37 \%$ phosphoric acid. ${ }^{35}$ This sum of factors possibly favors the opening of dentinal tubules to facilitate luting agent adhesion to dentin. Niu et al. when the canal is irrigated with $15 \%$ EDTA solution followed by $6 \% \mathrm{NaOCl}$, dentin presents an eroded aspect, with more irregular and rough dentinal tubules, favoring adhesion to dentin. ${ }^{36}$

Although studies showed that $2.5 \% \mathrm{NaOCl}$ and $17 \%$ EDTA have displayed the best values of bond strengths..$^{37,38} \mathrm{~A}$ study by Santos, et al. reported reduced bond strength values to a self-etching adhesive systems when the canals were irrigated using $5.25 \% \mathrm{NaOCl}$ combined with $17 \%$ EDTA compared to the sole use of $\mathrm{NaOCl}$ on dentin. ${ }^{30}$ This discrepancy might be related to the absence or presence of a smear layer. Moreover, Santos et al. used bovine incisors in their study while we chose to use sound human molars with total etch adhesive protocol in our study which could justify the disparity. When subsequent coronal sealing is performed, the use of $\mathrm{NaOCl}$ as a root canal irrigant, even if combined with EDTA, should be cautiously assessed..$^{39}$

In groups bonded with universal adhesive, adhesion after irrigation with EDTA or its combination with $\mathrm{NaOCL}$ resulted in significant improvement in the bond strength to dentin. The application of EDTA provided smear layer removal and create demineralized dentin zones, thus allowing greater interaction of the adhesive with dentin and enabling adhesive maintenance. ${ }^{40}$ EDTA as a chelating agent changes the surface topography by increasing surface roughness thereby increasing dentin wettability and the bond strength. ${ }^{41}$ SEM results confirm the results of the $\mu$ TBS where true hybridization with scanty short resin tag.

Niu et al. reported that the use of a 15\% EDTA solution followed by $6 \% \mathrm{NaOCl}$ as canal irrigants, could result in dentin erosion with rougher and less regular dentinal tubules enhancing dentin adhesion. ${ }^{36}$ However, a previous study showed that, there was no significant difference in the bond strengths of a self-etching adhesive system to group irrigated with a $1 \% \mathrm{NaOCl}$ solution followed by $17 \%$ EDTA and 
the control group where no irrigant was used. This implied that the dentin surface erosion caused by the use of EDTA might not have much effect on the dentin-resin bonding. In 2006, a study revealed that the employment of a combination of $5.25 \% \mathrm{NaOCl}$ and $17 \%$ EDTA as canal irrigants resulted in inferior bond strength values to a self-etching adhesive when compared to the use of $\mathrm{NaOCl}$ alone. ${ }^{30}$

From the results of the present study, it was found that universal adhesive could improve the bond strength in comparison to the total-etch adhesive regardless of the endodontic irrigants used as surface treatment for dentin. Superior performance of the universal adhesive can be attributed to the ethanol solvent incorporated in it. In demineralized dentin, ethanol could substitute water, fill in spaces between collagen fibers and increase the collagen matrix hydrophobicity in addition to being a solvent to most hydrophobic resin monomers. Accordingly, the penetration of such monomers into dentin collagen fibers and dentinal tubules is improved aiding the infiltration of hydrophobic resin to form a hybrid layer that is dense and homogenous for superior bonding. ${ }^{42,43}$ The use of the later ethanolwet bonding technique was associated with reduced nano-leakage. ${ }^{44}$

Based on the results of this study, our null hypothesis is totally rejected.

\section{CONCLUSIONS}

Based on the limitation of the present study; the following conclusions can be reported:

1- Application of combination of the endodontic irrigants, sodium hypochlorite and EDTA, was found to highly improve the quality of the universal adhesive bond to dentin.

2- The use of universal adhesive in a self-etch mode was found to result in superior values regarding bond strength when compared to total-etch adhesive.

3- Use of a combination of irrigants as a conditioner before adhesion protocol is recommended.

\section{RECOMMENDATIONS}

Further studies are required to investigate the effect of each mode of application of the universal adhesive after endodontic irrigation and the effects of endodontic irrigants on the bond strength of totaletch adhesive systems should be conducted.

\section{REFERENCES}

1. Nikaido T, Takano Y, Sasafuchi Y, et al. Bond strengths to endodontically-treated teeth. Am J Dent 1999; 12: 177-180.

2. Mohammadi Z, Yaripour S, Shalavi S, et al. Root Canal Irrigants and Dentin Bonding: An Update. Iran Endod J 2017; 12: 131-136.

3. Sayin TC, Serper A, Cehreli ZC, et al. The effect of EDTA, EGTA, EDTAC, and tetracycline-HCl with and without subsequent $\mathrm{NaOCl}$ treatment on the microhardness of root canal dentin. Oral Surg Oral Med Oral Pathol Oral Radiol Endod 2007; 104: 418-424.

4. Bosaid F, Aksel H, Makowka S, et al. Surface and structural changes in root dentine by various chelating solutions used in regenerative endodontics. Int Endod J 2020; 53 : $1438-1445$.

5. Soares JA, Roque de Carvalho MA, Cunha Santos SM, et al. Effectiveness of chemomechanical preparation with alternating use of sodium hypochlorite and EDTA in eliminating intracanal Enterococcus faecalis biofilm. J Endod 2010; 36: 894-898.

6. Nishitani Y, Yoshiyama M, Donnelly AM, et al. Effects of resin hydrophilicity on dentin bond strength. J Dent Res 2006; 85: 1016-1021.

7. Sismanoglu S, Ercal P. The cytotoxic effects of various endodontic irrigants on the viability of dental mesenchymal stem cells. Aust Endod J. Epub ahead of print 29 September 2021. DOI: 10.1111/aej.12570.

8. Soares CJ, Versluis A, Tantbirojn D, et al. Effect of Endodontic Treatment Procedures on Canal Shape and Mechanical Properties of a Tooth. In: Perdigão J (ed) Restoration of Root Canal-Treated Teeth: An Adhesive Dentistry Perspective. Cham: Springer International Publishing, 2016, pp. 25-43.

9. Amato M, Pantaleo G, Abtellatif D, et al. An in vitro evaluation of the degree of pulp tissue dissolution through different root canal irrigation protocols. J Conserv Dent 2018; 21: 175-179. 
10. Caron G, Nham K, Bronnec F, et al. Effectiveness of different final irrigant activation protocols on smear layer removal in curved canals. J Endod 2010; 36: 1361-1366.

11. Abo-Hamar SE. Effect of endodontic irrigation and dressing procedures on the shear bond strength of composite to coronal dentin. J Advert Res 2013; 4: 61-67.

12. Zhang K, Tay FR, Kim YK, et al. The effect of initial irrigation with two different sodium hypochlorite concentrations on the erosion of instrumented radicular dentin. Dent Mater 2010; 26: 514-523.

13. Kishen A, Sum C-P, Mathew S, et al. Influence of irrigation regimens on the adherence of Enterococcus faecalis to root canal dentin. J Endod 2008; 34: 850-854.

14. Faria ACL, Rodrigues RCS, de Almeida Antunes RP, et al. Endodontically treated teeth: characteristics and considerations to restore them. J Prosthodont Res 2011; 55: 69-74.

15. Peroz I, Blankenstein F, Lange K-P, et al. Restoring endodontically treated teeth with posts and cores--a review. Quintessence Int 2005; 36: 737-746.

16. Akkayan B, Gülmez T. Resistance to fracture of endodontically treated teeth restored with different post systems. J Prosthet Dent 2002; 87: 431-437.

17. Prasansuttiporn T, Nakajima M, Kunawarote S, et al. Effect of reducing agents on bond strength to NaOCl-treated dentin. Dent Mater 2011; 27: 229-234.

18. 18. Lai SC, Mak YF, Cheung GS, et al. Reversal of compromised bonding to oxidized etched dentin. J Dent Res 2001; 80: 1919-1924.

19. Ozturk B, Ozer F. Effect of $\mathrm{NaOCl}$ on bond strengths of bonding agents to pulp chamber lateral walls. J Endod 2004; 30: 362-365.

20. Cecchin D, Farina AP, Galafassi D, et al. Influence of sodium hypochlorite and edta on the microtensile bond strength of a self-etching adhesive system. J Appl Oral Sci 2010; 18: 385-389.

21. Pashley DH, Carvalho RM, Sano H, et al. The microtensile bond test: a review. J Adhes Dent 1999; 1: 299-309.

22. Dogan Buzoglu H, Calt S, Gümüsderelioglu M. Evaluation of the surface free energy on root canal dentine walls treated with chelating agents and $\mathrm{NaOCl}$. Int Endod $\mathrm{J}$ 2007; 40: 18-24.

23. Akyuz Ekim SN, Erdemir A. Comparison of different irrigation activation techniques on smear layer removal: an in vitro study. Microsc Res Tech 2015; 78: 230-239.
24. Perdigão J, Lopes M, Geraldeli S, et al. Effect of a sodium hypochlorite gel on dentin bonding. Dent Mater 2000; 16 : 311-323.

25. Pimenta L-AF, Amaral CM, Bedran de Castro AKB, et al. Stability of dentin bond strengths using different bonding techniques after 12 months: total-etch, deproteinization and self-etching. Oper Dent 2004; 29: 592-598.

26. Barbosa de Souza F, Silva CHV, Guenka Palma Dibb R, et al. Bonding performance of different adhesive systems to deproteinized dentin: microtensile bond strength and scanning electron microscopy. J Biomed Mater Res B Appl Biomater 2005; 75: 158-167.

27. Marending M, Paqué F, Fischer J, et al. Impact of irrigant sequence on mechanical properties of human root dentin. $\mathrm{J}$ Endod 2007; 33: 1325-1328.

28. Oliveira LD, Carvalho CAT, Nunes W, et al. Effects of chlorhexidine and sodium hypochlorite on the microhardness of root canal dentin. Oral Surg Oral Med Oral Pathol Oral Radiol Endod 2007; 104: e125-8.

29. Erdemir A, Ari H, Güngüneş H, et al. Effect of medications for root canal treatment on bonding to root canal dentin. $\mathrm{J}$ Endod 2004; 30: 113-116.

30. Santos JN, Carrilho MR de O, De Goes MF, et al. Effect of chemical irrigants on the bond strength of a self-etching adhesive to pulp chamber dentin. J Endod 2006; 32 : 1088-1090.

31. Osorio R, Erhardt MCG, Pimenta LAF, et al. EDTA treatment improves resin-dentin bonds' resistance to degradation. J Dent Res 2005; 84: 736-740.

32. Barón M, Llena C, Forner L, et al. Nanostructural changes in dentine caused by endodontic irrigants. Med Oral Patol Oral Cir Bucal 2013; 18: e733-6.

33. Kfir A, Goldenberg C, Metzger Z, et al. Cleanliness and erosion of root canal walls after irrigation with a new HEDP-based solution vs. traditional sodium hypochlorite followed by EDTA. A scanning electron microscope study. Clin Oral Investig 2020; 24: 3699-3706.

34. Wattanawongpitak N, Nakajima M, Ikeda M, et al. Microtensile bond strength of etch-and-rinse and self-etching adhesives to intrapulpal dentin after endodontic irrigation and setting of root canal sealer. J Adhes Dent 2009; 11: 57-64.

35. Radovic I, Mazzitelli C, Chieffi N, et al. Evaluation of the adhesion of fiber posts cemented using different adhesive approaches. Eur J Oral Sci 2008; 116: 557-563. 
36. Niu W, Yoshioka T, Kobayashi C, et al. A scanning electron microscopic study of dentinal erosion by final irrigation with EDTA and $\mathrm{NaOCl}$ solutions. Int Endod J 2002; 35: 934-939.

37. Galafassi D, Colucci V, Cecchin D, et al. Effect of endodontic irrigants on microtensile bond strength to dentin after thermocycling and long-term water storage. J Dent 2013; 10: 426-435.

38. Farina AP, Cecchin D, Barbizam JVB, et al. Influence of endodontic irrigants on bond strength of a self-etching adhesive. Aust Endod J 2011; 37: 26-30.

39. Barutcigil Ç, Harorlı OT, Özcan E, et al. Effects of ethylenediaminetetraacetic acid and sodium hypochlorite on the bond strength of bonding agents to pulp chamber lateral walls. Journal of Dental Sciences 2014; 9: 229-234.

40. García-Godoy F, Loushine RJ, Itthagarun A, et al. Application of biologically-oriented dentin bonding principles to the use of endodontic irrigants. Am J Dent 2005; 18 : 281-290.

41. Tartari T, Duarte Junior AP, Silva Júnior JOC, et al. Etidronate from medicine to endodontics: effects of different irrigation regimes on root dentin roughness. J Appl Oral Sci 2013; 21: 409-415.

42. Ahn J, Jung K-H, Son S-A, et al. Effect of additional etching and ethanol-wet bonding on the dentin bond strength of one-step self-etch adhesives. Restorative Dentistry \& Endodontics 2015; 40: 68.

43. Yang H, Guo J, Deng D, et al. Effect of adjunctive application of epigallocatechin-3-gallate and ethanol-wet bonding on adhesive-dentin bonds. Journal of Dentistry 2016; 44: 44-49.

44. Kuhn E, Farhat P, Teitelbaum AP, et al. Ethanol-wet bonding technique: Clinical versus laboratory findings. Dental Materials 2015; 31: 1030-1037. 